\title{
Gli autori di questo numero
}

\section{Guido Alpa}

Professore ordinario di Diritto civile nell'Università "La Sapienza" di Roma

Clara Benevolo

Professore associato in Economia e gestione delle imprese nell'Università di Genova

\section{Daniele Binci}

$\mathrm{PhD}$ in Economia ed organizzazione delle imprese nell'Università "Tor Vergata" di Roma

\section{Virginia Dagostino}

Collaboratore presso il Dipartimento di Economia e Metodi Quantitativi nell'Università di Genova

\section{Daniele Dalli}

Professore ordinario di Economia e gestione delle imprese nell’Università di Pisa

Enrico Di Taranto

Dottorando in Scienze del turismo ad indirizzo manageriale nell'Università "Federico II" di Napoli

\section{Giovanni Di Trapani}

Ricercatore presso l'Irat-Cnr di Napoli

Luisa Errichiello

Ricercatore presso l'Irat-Cnr di Napoli

\section{Mario Grasso}

Docente in Economia e gestione delle imprese turistiche nell'Università di Genova

\section{Giulio Maggiore}

Ricercatore in Economia e gestione delle imprese presso l'Università Telematica Unitelma Sapienza di Roma

\section{Renato Passaro}

Professore ordinario di Economia aziendale nell'Università "Parthenope" di Napoli

\section{Riccardo Resciniti}

Professore straordinario di Economia e gestione delle imprese nell'Università del Sannio

\section{Ruggero Sainaghi}

Ricercatore confermato nell'Università IULM di Milano e idoneo come professore di seconda fascia

\section{Antonio Thomas}

Ricercatore in Economia aziendale nell'Università "Parthenope" di Napoli

\section{Annalisa Tunisini}

Professore ordinario di Economia e gestione delle imprese nell'Università "Carlo Bo" di Urbino

\section{Riccardo Varaldo}

Professore ordinario di Economia e gestione delle imprese e Presidente della Scuola Superiore Sant'Anna di Pisa 